\title{
Seurat: A Pointillist Approach to Anomaly Detection
}

\author{
Yinglian Xie ${ }^{1}$, Hyang-Ah Kim ${ }^{1}$ \\ David R. O’Hallaron ${ }^{12}$, Michael K. Reiter ${ }^{12}$, and Hui Zhang ${ }^{12}$ \\ 1 Department of Computer Science \\ 2 Department of Electrical and Computer Engineering \\ Carnegie Mellon University \\ Email: \{ylxie, hakim, droh, reiter, hzhang\}@cs.cmu.edu
}

\begin{abstract}
This paper proposes a new approach to detecting aggregated anomalous events by correlating host file system changes across space and time. Our approach is based on a key observation that many host state transitions of interest have both temporal and spatial locality. Abnormal state changes, which may be hard to detect in isolation, become apparent when they are correlated with similar changes on other hosts. Based on this intuition, we have developed a method to detect similar, coincident changes to the patterns of file updates that are shared across multiple hosts. We have implemented this approach in a prototype system called Seurat and demonstrated its effectiveness using a combination of real workstation cluster traces, simulated attacks, and a manually launched Linux worm.
\end{abstract}

Keywords: Anomaly detection, Pointillism, Correlation, File updates, Clustering

\section{Introduction}

Correlation is a recognized technique for improving the effectiveness of intrusion detection by combining information from multiple sources. For example, many existing works have proposed correlating different types of logs gathered from distributed measurement points on a network (e.g., [1-3]). By leveraging collective information from different local detection systems, they are able to detect more attacks with fewer false positives.

In this paper, we propose a new approach to anomaly detection based on the idea of correlating host state transitions such as fi le system updates. The idea is to correlate host state transitions across both space (multiple hosts) and time (the past and the present), detecting similar coincident changes to the patterns of host state updates that are shared across multiple hosts. Examples of such coincident events include administrative updates that modify fi les that have not been modifi ed before, and malware propagations that cause certain log fi les, which are modifi ed daily, to cease being updated.

Our approach is based on the key observation that changes in host state in a network system often have both temporal and spatial locality. Both administrative updates and malware propagation exhibit spatial locality, in the sense that similar updates tend to occur across many of the hosts in a network. They also exhibit temporal locality in the 
sense that these updates tend to be clustered closely in time. Our goal is to identify atypical such aggregate updates, or the lack of typical ones.

By exploring both the temporal and spatial locality of host state changes in a network system, our approach identifi es anomalies without foreknowledge of normal changes and without system-specific knowledge. Existing approaches focus on the temporal locality of host state transitions, while overlooking the spatial locality among different hosts in a network system. They either defi ne a model of normal host state change patterns through learning, or specify detailed rules about normal changes. The learning based approaches train the system to learn characteristics of normal changes. Since they focus only on the temporal locality of single-host state transitions, any signifi cant deviation from the normal model is suspicious and should raise an alarm, resulting in a high false positive rate. Rule-based approaches such as Tripwire [4] require accurate, specifi c knowledge of system confi gurations and daily user activity patterns on a specifi c host. Violation of rules then suggests malicious intrusions. Although rule-based anomaly detection raises fewer false alarms, it requires system administrators to manually specify a set of rules for each host. The correlation capability of our approach across both space and time allows us to learn the patterns of normal state changes over time, and to detect those anomalous events correlated among multiple hosts due to malicious intrusions. This obviates the need for specifi $\mathrm{c}$ rules while eliminating the false alarms caused by single host activity pattern shifts.

The correlation is performed by clustering points, each representing an individual host state transition, in a multi-dimensional feature space. Each feature indicates the change of a fi le attribute, with all features together describing the host state transitions of an individual machine during a given period (e.g., one day). Over time, the abstraction of point patterns inherently reflects the aggregated host activities. For normal host state changes, the points should follow some regular pattern by roughly falling into several clusters. Abnormal changes, which are hard to detect by monitoring that host alone, will stand out when they are correlated with other normal host state changes. Hence our approach shares some flavor of pointillism - a style of painting that applies small dots onto a surface so that from a distance the dots blend together into meaningful patterns.

Figure 1 illustrates the pointillist approach to anomaly detection. There are five hosts in the network system. We represent state changes on each host daily as a point in a 2-dimensional space in this example. On normal days, the points roughly fall into the dash-circled region. The appearance of a new cluster consisting of three points (indicated by the solid circle) suggests the incidence of anomaly on host A, B, and D, which may all have been compromised by the same attack. Furthermore, if we know that certain hosts (e.g., host A) are already compromised (possibly detected by other means such as a network based IDS), then we can correlate the state changes of the compromised hosts with the state changes of all other hosts in the network system to detect more infected hosts (e.g., host B and D).

We have implemented a prototype system, called Seurat ${ }^{3}$, that uses fi le system updates to represent host state changes for anomaly detection. Seurat successfully detects the propagation of a manually launched Linux worm on a number of hosts in an isolated cluster. Seurat has a low false alarm rate when evaluated by a real deployment. These

\footnotetext{
${ }^{3}$ Seurat is the 19 th century founder of pointillism.
} 


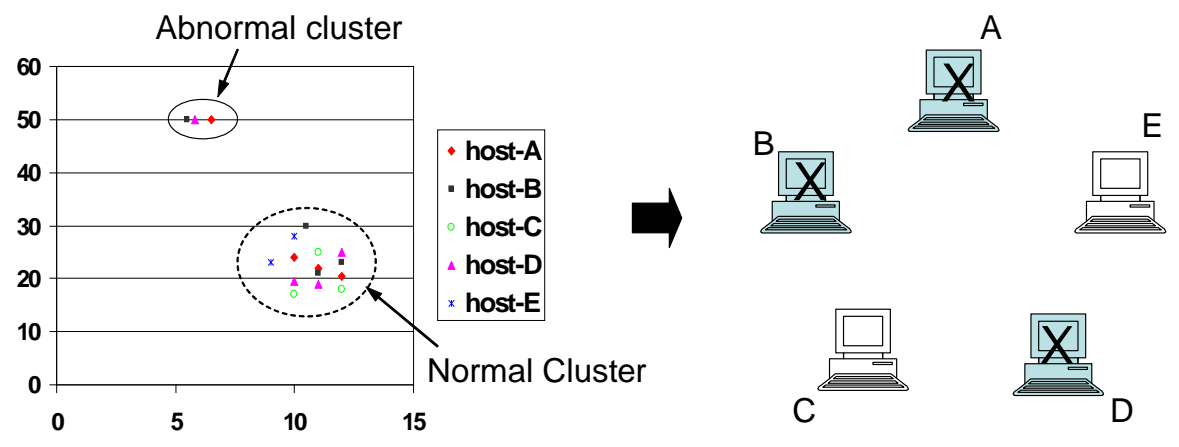

Fig. 1. Pointillist approach to anomaly detection: Normal points are clustered by the dashed circle. The appearance of a new cluster consisting of three points suggests anomalous events on host A, B, and D.

alarms are caused by either system re-confi gurations or network wide experiments. The false negative rate and detection latency, evaluated with simulated attacks, are both low for fast propagating attacks. For slowly propagating attacks, there is a tradeoff between false negative rate and detection latency. For each alarm, Seurat identifi es the list of hosts involved and the related fi les, which we expect will be extremely helpful for system administrators to examine the root cause and dismiss false alarms.

The rest of the paper is organized as follows: Section 2 describes Seurat threat model. Section 3 introduces the algorithm for correlating host state changes across both space and time. Section 4 evaluates our approach. Section 5 discusses the limitations of Seurat and suggests possible improvements. Section 6 presents related work.

\section{Attack Model}

The goal of Seurat is to automatically identify anomalous events by correlating the state change events of all hosts in a network system. Hence Seurat defi nes an anomalous event as an unexpected state change close in time across multiple hosts in a network system.

We focus on rapidly propagating Internet worms, virus, zombies, or other malicious attacks that compromise multiple hosts in a network system at a time (e.g., one or two days). We have observed that, once fast, automated attacks are launched, most of the vulnerable hosts get compromised due to the rapid propagation of the attack and the scanning preferences of the automated attack tools. According to CERT's analysis [5], the level of automation in attack tools continues to increase, making it faster to search vulnerable hosts and propagate attacks. Recently, the Slammer worm hit 90 percent of vulnerable systems in the Internet within 10 minutes [6]. Worse, the lack of diversity in systems and softwares run by Internet-attached hosts enables massive and fast attacks. Computer clusters tend to be confi gured with the same operating systems and softwares. In such systems, host state changes due to attacks have temporal and spatial locality that can be exploited by Seurat. 
Although Seurat is originally designed to detect system changes due to fast propagating attacks, it can be generalized to detect slowly propagating attacks as well. This can be done by varying the time resolution of reporting and correlating the collective host state changes. We will discuss this issue further in Section 5. However, Seurat's global correlation can not detect abnormal state changes that are unique to only a single host in the network system.

Seurat represents host state changes using file system updates. Pennington et al. [7] found that $83 \%$ of the intrusion tools and network worms they surveyed modify one or more system fi les. These modifi cations would be noticed by monitoring fi le system updates. There are many security tools such as Tripwire [4] and AIDE [8] that rely on monitoring abnormal fi le system updates for intrusion detection.

We use the file name, including its complete path, to identify a file in the network system. We regard different instances of a fi le that correspond to a common path name as a same fi le across different hosts, since we are mostly interested in system fi les which tend to have canonical path names exploited by malicious attacks. We treat fi les with different path names on different hosts as different fi les, even when they are identical in content.

For the detection of anomalies caused by attacks, we have found that this representation of host state changes is effective and useful. However, we may need different approaches for other applications of Seurat such as fi le sharing detection, or for the detection of more sophisticated future attacks that alter fi les at arbitrary locations as they propagate. As ongoing work, we are investigating the use of fi le content digests instead of fi le names.

\section{Correlation-based Anomaly Detection}

We defi ne a $d$-dimensional feature vector $\boldsymbol{H}_{i j}=\left\langle v_{1}, v_{2}, \ldots, v_{d}\right\rangle$ to represent the fi le system update attributes for host $i$ during time period $j$. Each $\boldsymbol{H}_{i j}$ can be plotted as a point in a $d$-dimensional feature space. Our pointillist approach is based on correlating the feature vectors by clustering. Over time, for normal fi le updates, the points follow some regular pattern (e.g., roughly fall into several clusters). From time to time, Seurat compares the newly generated points against points from previous time periods. The appearance of a new cluster, consisting only of newly generated points, indicates abnormal fi le updates and Seurat raises an alarm.

For clustering to work most effectively, we need to fi nd the most relevant features (dimensions) in a feature vector given all the fi le update attributes collected by Seurat. We have investigated two methods to reduce the feature vector dimensions: (1) waveletbased selection, and (2) principal component analysis (PCA).

In the rest of this section, we first present how we defi ne the feature vector space and the distances among points. We then describe the methods Seurat uses to reduce

feature vector dimensions. Finally, we discuss how Seurat detects abnormal fi le updates by clustering. 


\subsection{Feature Vector Space}

Seurat uses binary feature vectors to represent host fi le updates. Each dimension in the feature vector space corresponds to a unique fi le (indexed by the full-path fi le name). As such, the dimension of the space $d$ is the number of fi le names present on any machine in the network system. We defi ne the detection window to be the period that we are interested in fi nding anomalies. In the current prototype, the detection window is one day. For each vector $\boldsymbol{H}_{i j}=\left\langle v_{1}, v_{2}, \ldots, v_{d}\right\rangle$, we set $v_{k}$ to 1 if host $i$ has updated (added, modifi ed, or removed) the $k$-th fi le on day $j$, otherwise, we set $\psi$ to 0 .

The vectors generated in the detection window will be correlated with vectors generated on multiple previous days. We treat each feature vector as an independent point in a set. The set can include vectors generated by the same host on multiple days, or vectors generated by multiple hosts on the same day. In the rest of the paper, we use $\boldsymbol{V}=\left\langle v_{1}, v_{2}, \ldots, v_{d}\right\rangle$ to denote a feature vector for convenience. Figure 2 shows how we represent the host fi le updates using feature vectors.

\begin{tabular}{|c|c|c|c|c|c|c|}
\hline & & $F_{1}$ & $\mathrm{~F}_{2}$ & $F_{3}$ & $\mathrm{~F}_{4}$ & $\mathrm{~F}_{5}$ \\
\hline $\mathrm{V}_{1}=\mathrm{H}_{11}=$ & & 1 & 1 & 0 & 1 & 1 \\
\hline $\mathrm{V}_{2}=\mathrm{H}_{21}=$ & & 1 , & 1 & 1 & 0 & 0 \\
\hline $\mathrm{V}_{3}=\mathrm{H}_{12}=$ & & & 1 & 0 & 1 & 0 \\
\hline
\end{tabular}

Fig. 2. Representing host file updates as feature vectors: $F_{1}, F_{2}, F_{3}, F_{4}, F_{5}$ are five different files (i.e., file names). Accordingly, the feature vector space has 5 dimensions in the example.

The correlation is based on the distances among vectors. Seurat uses a cosine distance metric, which is a common similarity measure between binary vectors $[9,10]$. We defi ne the distance $D\left(\boldsymbol{V}_{1}, \boldsymbol{V}_{2}\right)$ between two vectors $\boldsymbol{V}_{1}$ and $\boldsymbol{V}_{2}$ as their angle $\theta$ computed by the cosine value:

$$
D\left(\boldsymbol{V}_{1}, \boldsymbol{V}_{2}\right)=\theta=\cos ^{-1}\left(\frac{\boldsymbol{V}_{1} \cdot \boldsymbol{V}_{2}}{\left|\boldsymbol{V}_{1}\right|\left|\boldsymbol{V}_{2}\right|}\right)
$$

For each day $j$ (the detection window), Seurat correlates the newly generated vectors with vectors generated in a number of previous days $j-1, j-2, \ldots$ We assume that the same abnormal fi le update events on day $j$, if any, have not occurred on those previous days. We defi ne the comparison window of day $j$ as the days that we look back for comparison, and the correlation window of day $j$ as the inclusive period of day $j$ and its comparison window. Vectors generated outside the correlation window of day $j$ are not used to identify abnormal fi le updates on day $j$. Figure 3 illustrates the concepts of detection window, comparison window, and correlation window.

Since each vector generated during the comparison window serves as an example of normal fi le updates to compare against in the clustering process, we explore the temporal locality of normal update events by choosing an appropriate comparison window for 


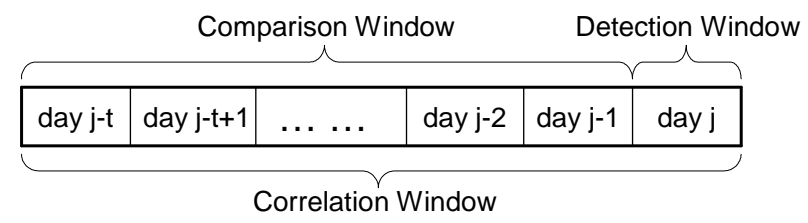

Fig. 3. Detection window, comparison window, and correlation window. The detection window is day $j$. The comparison window is from day $j-t$ to day $j-1$. The correlation window is from day $j-t$ to day $j$.

each day. The comparison window size is a confi gurable parameter of Seurat. It reflects how far we look back into history to implicitly defi ne the model of normal fi le updates. For example, some fi les such as /var/spool/anacron/cron. weekly on Linux platforms are updated weekly. In order to regard such weekly updates as normal updates, administrators have to choose a comparison window size larger than a week. Similarly, the size of the detection window reflects the degree of temporal locality of abnormal update events.

Since Seurat correlates fi le updates across multiple hosts, we are interested in only those fi les that have been updated by at least two different hosts. Files that have been updated by only one single host in the network system throughout the correlation window are more likely to be user fi les. As such, we do not select them as relevant dimensions to defi ne the feature vector space.

\subsection{Feature Selection}

Most fi le updates are irrelevant to anomalous events even after we fi lter out the fi le updates reported by a single host. Those fi les become noise dimensions when we correlate the vectors (points) to identify abnormal updates, and increase the complexity of the correlation process. We need more selective ways to choose relevant fi les and reduce feature vector dimensions. Seurat uses a wavelet-based selection method and principal component analysis (PCA) for this purpose.

Wavelet-based Selection The wavelet-based selection method regards each individual fi le update status as a discrete time series signal $S$. Given a fi le $i$, the value of the signal on day $n$, denoted by $S_{i}(n)$, is defi ned as the total number of hosts that update fi le $i$ on day $n$ in the network system. Each such signal $S_{i}$ can be decomposed into a low frequency signal $c A_{i}$ reflecting the long term update trend, and a high frequency signal $c D_{i}$ reflecting the day-to-day variation from the long term trend. (see Figure 4). If the high frequency signal $c A_{i}$ shows a spike on a certain day, we know that a signifi cantly larger number of hosts updated file $i$ than on a normal day. We then select fi le $i$ as a relevant feature dimension in defi ning the feature vector space.

Seurat detects signal spikes using the residual signal of the long-term trend. The same technique has been used to detect disease outbreaks[11]. To detect anomalies on day $j$, the algorithm takes as input the list of fi les that have been updated by at least two 


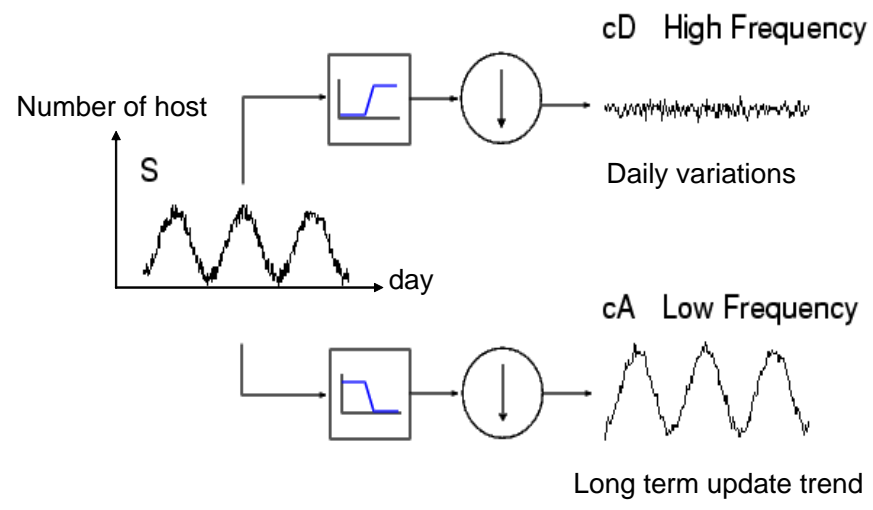

Fig. 4. Representing file update status with wavelet transformation: The original signal is $S$, which can be decomposed into a low frequency signal $c A$ reffecting the long term update trend, and a high frequency signal $c D$ refecting the daily variations from the long-term trend.

different hosts in the correlation window of day $j$. Then, from these fi les the algorithm selects a subset that will be used to defi ne the feature vector space.

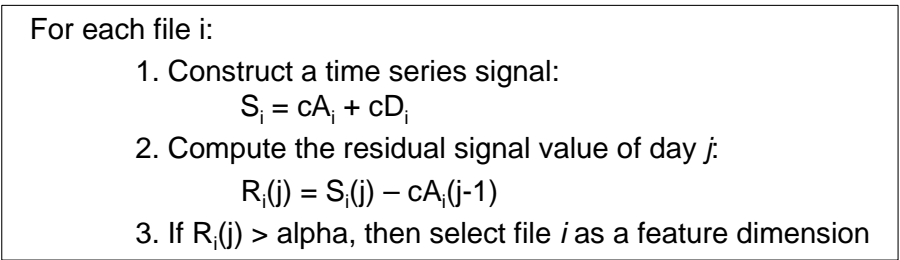

Fig. 5. Wavelet-based feature selection

Figure 5 shows the steps to select features by wavelet-based method. Given a fi xed correlation window of day $j$, the algorithm starts with constructing a time series signal $S_{i}$ for each fi le $i$, and decomposes $S_{i}$ into $c A_{i}$ and $c D_{i}$ using a single-level wavelet transformation as described. Then we compute the residual signal value $R_{i}(j)$ of day $j$ by subtracting the trend value $c A_{i}(j-1)$ of day $j-1$ from the original signal value $S_{i}(j)$ of day $j$. If $R_{i}(j)$ exceeds a pre-set threshold $\alpha$, then the actual number of hosts who have updated fi le $i$ on day $j$ is signifi cantly larger than the prediction $c A(j-1)$ based on the long term trend. Therefore, Seurat selects fi le $i$ as an interesting feature dimension for anomaly detection on day $j$. As an example, Figure 6 shows the original signal and the residual signal of a fi le using a 32-day correlation window in a 22-host teaching cluster. Note the threshold value $\alpha$ of each fi le is a parameter selected based on the statistical distribution of historical residual values. 


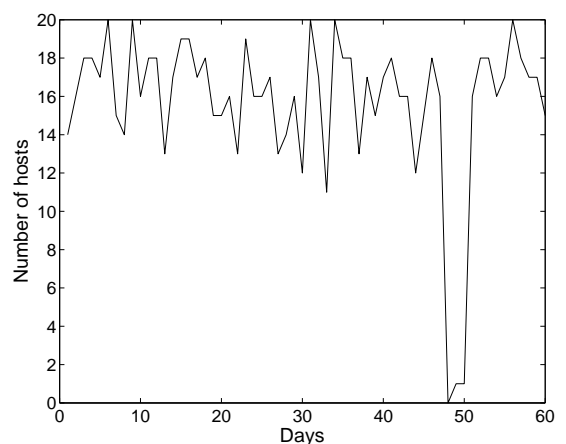

(a)

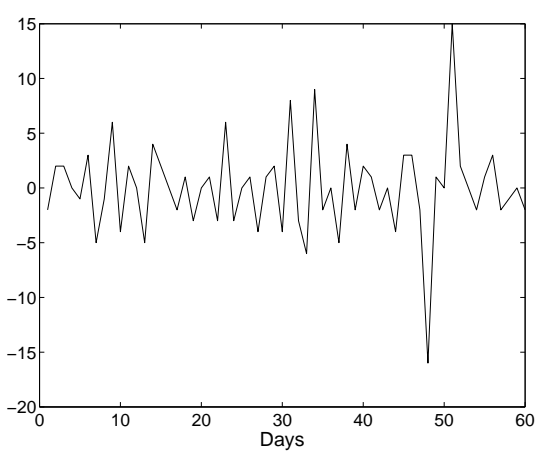

(b)

Fig. 6. Wavelet transformation of file update status: (a) The original signal of the file update status (b) The residual signal after wavelet transformation

PCA-based Dimension Reduction PCA is a statistical method to reduce data dimensionality without much loss of information [12]. Given a set of $d$-dimensional data points, PCA fi nds a set of $d$ orthogonal vectors, called principal components, that account for the variance of the input data as much as possible. Dimensionality reduction is achieved by projecting the original $d$-dimensional data onto the subspace spanned by these $d^{\prime}$ orthogonal vectors. Most of the intrinsic information of the $d$-dimensional data is preserved in the $d^{\prime}$-dimensional subspace.

We note that the updates of different fi les are usually correlated. For example, when a software package is updated on a host, many of the related fi les will be modifi ed together. Thus we can perform PCA to identify the correlation of fi le updates.

Given a $d$-dimensional feature space $\mathcal{Z}_{2}^{d}$, and a list of $m$ feature vectors $\boldsymbol{V}_{1}, \boldsymbol{V}_{2}, \ldots$, $\boldsymbol{V}_{m} \in \mathcal{Z}_{2}^{d}$, we perform the following steps using PCA to obtain a new list of feature vectors $\boldsymbol{V}_{1}^{\prime}, \boldsymbol{V}_{2}^{\prime}, \ldots, \boldsymbol{V}_{m}^{\prime} \in \mathcal{Z}_{2}^{d^{\prime}}\left(d^{\prime}<d\right)$ with reduced number of dimensions:

1. Standardize each feature vector $\boldsymbol{V}_{k}=\left\langle v_{1 k}, v_{2 k}, \ldots, v_{d k}\right\rangle(1 \leq k \leq m)$ by subtracting each of its elements $v_{i k}$ by the mean value of the corresponding dimension $u_{i}(1 \leq i \leq d)$. We use $\overline{\boldsymbol{V}}_{\boldsymbol{k}}=\left\langle\bar{v}_{1 k}, \bar{v}_{2 k}, \ldots, \bar{v}_{n k}\right\rangle \in \mathcal{Z}_{2}^{d}$ to denote the standardized vector for the original feature vector $\boldsymbol{V}_{k}$. Then,

$$
\bar{v}_{i k}=v_{i k}-u_{i}\left(\text { where } u_{i}=\frac{\sum_{j=1}^{m} v_{i j}}{m}, 1 \leq i \leq d\right)
$$

2. Use the standardized feature vectors $\bar{V}_{1}, \bar{V}_{2}, \ldots, \bar{V}_{m}$ as input data to PCA in order to identify a set of principal components that are orthogonal vectors defi ning a set of transformed dimensions of the original feature space $\mathcal{Z}_{2}^{d}$. Select the fi rst $d$ principal components that count for most of the input data variances (e.g., $90 \%$ of data variances) to defi ne a subspace $\mathcal{Z}_{2}^{d^{\prime}}$.

3. Project each standardized feature vector $\overline{\boldsymbol{V}}_{\boldsymbol{k}} \in \mathcal{Z}_{2}^{d}$ onto the PCA selected subspace $\mathcal{Z}_{2}^{d^{\prime}}$ to obtain the corresponding reduced dimension vector $\boldsymbol{V}_{\boldsymbol{k}}^{\prime} \in \mathcal{Z}_{2}^{d^{\prime}}$. 
Note that PCA is complementary to wavelet-based selection. Once we fi $\mathrm{x}$ the correlation window of a particular day, we fi rst pick a set of fi les to defi ne the feature vector space by wavelet-based selection. We then perform PCA to reduce the data dimensionality further.

\subsection{Anomaly Detection by Clustering}

Once we obtain a list of transformed feature vectors using feature selection, we cluster the vectors based on the distance between every pair of them.

We call the cluster a new cluster if it consists of multiple vectors only from the detection window. The appearance of a new cluster indicates possibly abnormal fi le updates occurred during the detection window and should raise an alarm.

There are many existing algorithms for clustering, for example, K-means $[13,14]$ or Single Linkage Hierarchical Clustering [10]. Seurat uses a simple iterative algorithm, which is a common method for K-means initialization, to cluster vectors without prior knowledge of the number of clusters [15]. The algorithm assumes each cluster has a hub. A vector belongs to the cluster whose hub is closest to that vector compared with the distances from other hubs to that vector. The algorithm starts with one cluster whose hub is randomly chosen. Then, it iteratively selects a vector that has the largest distance to its own hub as a new hub, and re-clusters all the vectors based on their distances to all the selected hubs. This process continues until there is no vector whose distance to its hub is larger than the half of the average hub-hub distance.

We choose this simple iterative algorithm because it runs much faster, and works equally well as the Single Linkage Hierarchical algorithm in our experiments. The reason that even the simple clustering algorithm works well is that the ratio of inter-cluster distance to intra-cluster distance signifi cantly increases after feature selection. Since the current clustering algorithm is sensitive to outliers, we plan to explore other clustering algorithms such as K-means.

Once we detect a new cluster and generate an alarm, we examine further to identify the involved hosts and the files from which the cluster resulted. The suspicious hosts are just the ones whose fi le updates correspond to the feature vectors in the new cluster. To determine which fi les possibly cause the alarm, we only focus on the fi les picked by the wavelet-based selection to defi ne the feature vector space. For each of those fi les, if it is updated by all the hosts in the new cluster during the detection window, but has not been updated by any host during the corresponding comparison window, Seurat outputs this fi le as a candidate fi le. Similarly, Seurat also reports the set of fi les that have been updated during the comparison window, but are not updated by any host in the new cluster during the detection window.

Based on the suspicious hosts and the selected fi les for explaining root causes, system administrators can decide whether the updates are known administrative updates that should be suppressed, or some abnormal events that should be further investigated. If the updates are caused by malicious attacks, administrators can take remedial counter measures for the new cluster. Furthermore, additional compromised hosts can be identifi ed by checking if the new cluster expands later and if other hosts have updated the same set of candidate fi les. 


\section{Experiments}

We have developed a multi-platform (Linux and Windows) prototype of Seurat that consists of a lightweight data collection tool and a correlation module. The data collection tool scans the fi le system of the host where it is running and generates a daily summary of fi le update attributes. Seurat harvests the summary reports from multiple hosts in a network system and the correlation module uses the reports for anomaly detection.

We have installed the Seurat data collection tool on a number of campus offi ce machines and a teaching cluster that are used by students daily. By default, the tool scans the attributes of all system fi les on a host. For privacy reasons, personal fi les under user home directories are not scanned. The attributes of a fi le include the fi le name, type, device number, permissions, size, inode number, important timestamps, and a 16-byte MD5 checksum of fi le content. The current system uses only a binary bit to represent each fi le update, but the next version may exploit other attributes reported by the data collection tool. Each day, each host compares the newly scanned disk snapshot against that from the previous day and generates a fi le update summary report. In the current prototype, all the reports are uploaded daily to a centralized server where system administrators can monitor and correlate the fi le updates using the correlation module.

In this section, we study the effectiveness of Seurat's pointillist approach for detecting aggregated anomalous events. We use the daily fi le update reports from our real deployment to study the false positive rate and the corresponding causes in Section 4.1. We evaluate the false negative rate with simulated attacks in Section 4.2. In order to verify the effectiveness of our approach on real malicious attacks, we launched a real Linux worm into an isolated cluster and report the results in Section 4.3.

\subsection{False Positives}

The best way to study the effectiveness of our approach is to test it with real data. We have deployed Seurat on a teaching cluster of 22 hosts and have been collecting the daily fi le update reports since Nov 2003. The teaching cluster is mostly used by students for their programming assignments. They are also occasionally used by a few graduate students for running network experiments.

For this experiment, we use the fi le update reports from Dec 1, 2003 until Feb 29, 2004 to evaluate the false positive rate. During this period, there are a few days when a couple of hosts failed to generate or upload reports due to system failure or reconfi gurations. For those small number of missing reports, we simply ignore them because they do not affect the aggregated fi le update patterns.

We set the correlation window to 32 days in order to accommodate monthly fi le update patterns. That is, we correlate the update pattern from day 1 to day 32 to identify abnormal events on day 32 , and correlate the update pattern from day 2 to day 33 to detect anomalies on day 33, etc. Thus, our detection starts from Jan 1, 2004, since we do not have 32-day correlation windows for the days in Dec 2003.

Dimension Reduction Once we fi xed the correlation window of a particular day, we identify relevant fi les using wavelet-based selection with a constant threshold $\alpha=2$ to 
defi ne the feature vector space for simplicity. We then perform PCA to reduce the data dimensionality further by picking the fir rst several principal components that account for $98 \%$ of the input data variances.

Throughout the entire period of 91 days, $772 \mathrm{fi}$ les with unique fi le names were updated by at least two different hosts. Figure 7 (a) shows the number of hosts that updated each fi le during the data collection period. We observe that only a small number files (e.g.,/var/adm/syslog/mail.log) are updated regularly by all of the hosts, while most other fi les (e.g., /var/run/named.pid) are updated irregularly, depending on the system usage or the applications running.

Figure 7 (b) shows the results of feature selection. There were, on average, $140 \mathrm{fi}$ les updated by at least two different hosts during each correlation window. After waveletbased selection, the average number of feature dimensions is 17. PCA further reduces the vector space dimension to below 10 .

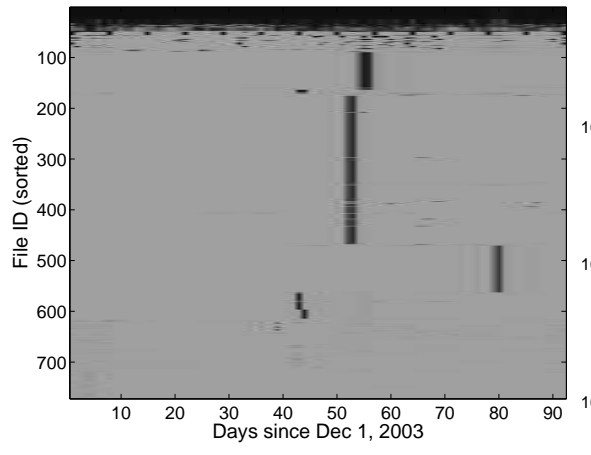

(a)

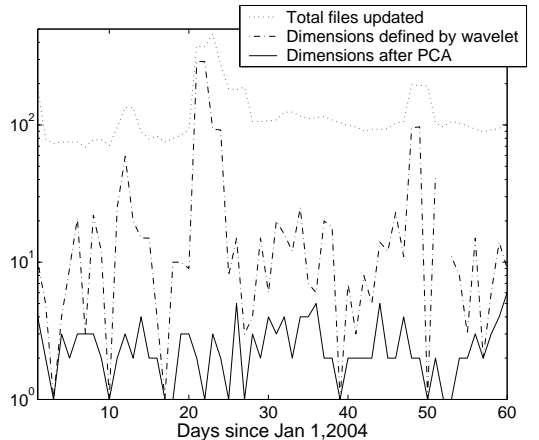

(b)

Fig. 7. Feature selection and dimension reduction: (a) File update patterns. Files are sorted by the cumulative number of hosts that have updated them throughout the 91 days. The darker the color is, the more hosts updated the corresponding file. (b) The number of feature vector dimensions after wavelet-based selection and PCA consecutively.

False Alarms After dimension reduction, we perform clustering of feature vectors and identify new clusters for each day. Figure 8 illustrates the clustering results of 6 consecutive days from Jan 19, 2004 to Jan 24, 2004. There are two new clusters identifi ed on Jan 21 and Jan 23, which involve 9 hosts and 6 hosts, respectively. Since Seurat outputs a list of suspicious fi les as the cause of each alarm, system administrators can tell if the new clusters are caused by malicious intrusions.

Based on the list of fi les output by Seurat, we can fi gure out that the new clusters on Jan 21 and Jan 23 reflect large scale fi le updates due to a system reconfi guration at the beginning of the spring semester. For both days, Seurat accurately pinpoints the exact hosts that are involved. The reconfi guration started from Jan 21, when a large number of binaries, header fi les, and library fi les were modifi ed on 9 out of the 22 hosts. Since 

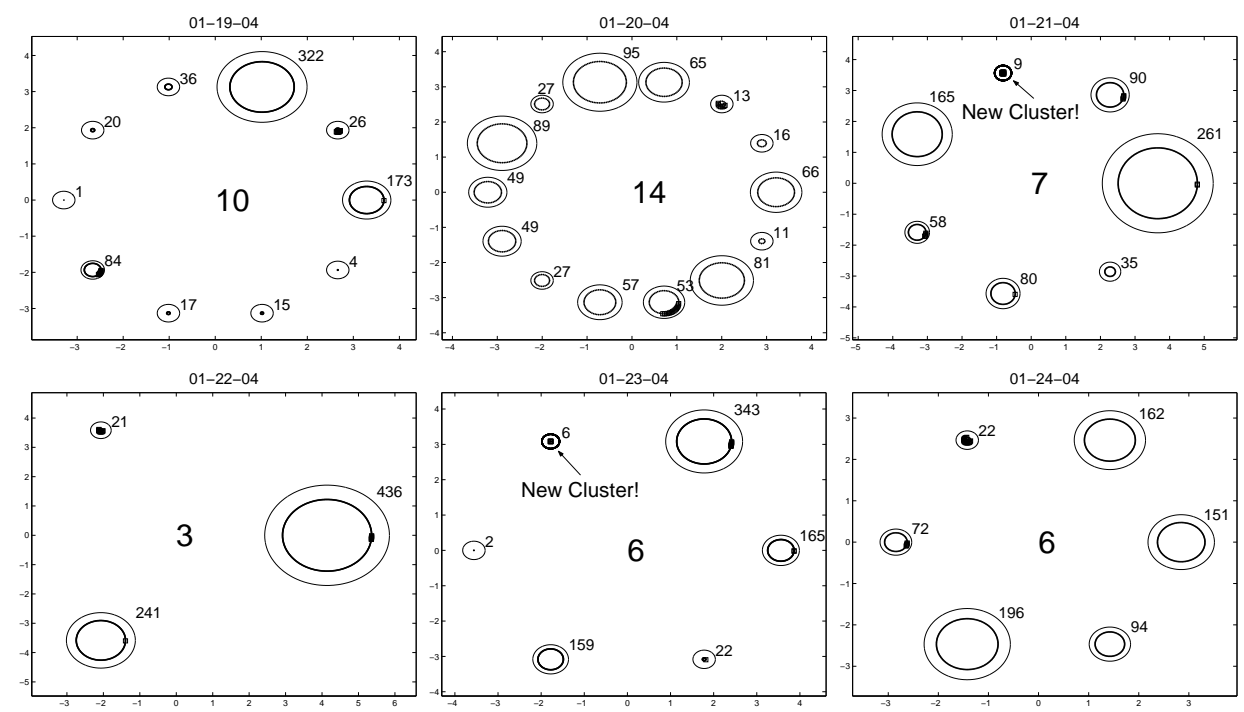

Fig. 8. Clustering feature vectors for anomaly detection: Each circle represents a cluster. The number at the center of the figure shows the total number of clusters. The radius of a circle corresponds to the number of points in the cluster, which is also indicated beside the circle. The squared dots correspond to the new points generated on the day under detection. New clusters are identified by a thicker circle.

the events are known to system administrators, we treat the identifi ed vectors as normal for future anomaly detection. Thus, no alarm is triggered on Jan 22, when the same set of library fi les were modifi ed on 12 other hosts. On Jan 23, the re-confi guration continued to remove a set of printer fi les on 6 out of the 22 hosts. Again, administrators can mark this event as normal and we spot no new cluster on Jan 24, when 14 other hosts underwent the same set of fi le updates.

In total, Seurat raises alarms on 9 out of the 60 days under detection, among which 6 were due to system re-confi gurations. Since the system administrators are aware of such events in advance, they can simply suppress these alarms. The 3 other alarms are generated on 3 consecutive days when a graduate student performed a network experiment that involved simultaneous fi le updates at multiple hosts. Such events are normal but rare, and should alert the system administrators.

\subsection{False Negatives}

The primary goal of this experiment is to study the false negative rate and detection latency of Seurat as the stealthiness of the attack changes. We use simulated attacks by manually updating fi les on the selected host reports, as if they were infected.

We fi rst examine the detection rate of Seurat by varying the degree of attack aggressiveness. We model the attack propagation speed as the number of hosts infected on each day (the detection window), and model the attack stealthiness on a local host 
as the number of new fi les installed by this attack. Our simulation runs on the same teaching cluster that we described in Section 4.1. Since the aggregated fi le update patterns are different for each day, we randomly pick ten days in Feb 2004, when there was no intrusion. On each selected day, we simulate attacks by manually inserting artifi cial new fi les into a number of host reports on only that day, and use the modifi ed reports as input for detection algorithm. We then remove those modifi ed entries, and repeat the experiments with another day. The detection rate is calculated as the number of days that Seurat spots new clusters over the total ten days.

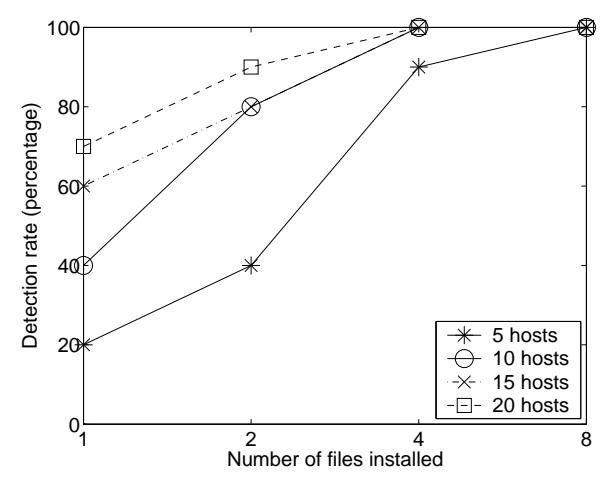

Fig. 9. Detection rate: We vary the number of hosts infected and the number of files inserted on each host by the simulated attacks.

Figure 9 shows the detection rate of Seurat by varying the number of fi les inserted on each host and the number of hosts infected. On one hand, the detection rate monotonically increases as we increase the number of fi les inserted on each host by an attack. Since the inserted fi les do not exist before, each of them will be selected as a feature dimension by the wavelet-based selection, leading to larger distances between the points of infected host state changes and the points of normal host state changes. Therefore, the more new fi les are injected by an attack, the higher the detection rate gets. On the other hand, as we increase the number of infected hosts, the number of points for abnormal host state changes becomes large enough to create an independent new cluster. Thus, rapidly propagating attacks are more likely to be caught. Accordingly, detecting a slowly propagating attack requires a larger detection window, hence longer detection latency, in order to accumulate enough infected hosts. We revisit this issue in Section 5.

We further evaluate the detection rate of Seurat on six Linux worms with simulated attacks. To do so, we compile a subset of fi les modifi ed by each worm based on the descriptions from public Web sites such as Symantec [16] and F-Secure information center [17]. We then manually modify the described fi les in a number of selected host reports to simulate the corresponding worm attacks. Again, for each worm, we vary the number of infected hosts, and run our experiments on the teaching cluster with ten randomly selected days. 


\begin{tabular}{|c||c|c|c|c|c|c|}
\hline Worms & Adore & Ramen-A & Ramen-B & Slapper-A & Slapper-B & Kork \\
\hline Files modified & 10 & 8 & 12 & 3 & 4 & 5 \\
\hline 2 infected hosts & $80 \%$ & $80 \%$ & $90 \%$ & $30 \%$ & $40 \%$ & $30 \%$ \\
\hline 4 infected hosts & $100 \%$ & $100 \%$ & $90 \%$ & $70 \%$ & $80 \%$ & $70 \%$ \\
\hline 8 infected hosts & $100 \%$ & $100 \%$ & $100 \%$ & $100 \%$ & $100 \%$ & $100 \%$ \\
\hline
\end{tabular}

Fig. 10. Detection rate of emulated worms: We vary the number of hosts compromised by the attacks.

Table 10 shows the number of fi les modifi ed by each worm and the detection rate of Seurat. In general, the more fi les modifi ed by a worm, the more likely the worm will be detected. But the position of a fi le in the fi le system directory tree also matters. For example, both Slapper-B worm and Kork worm insert 4 new fi les into a compromised host. However, Kork worm additionally modifi es /etc/passwd to create accounts with root privileges. Because there are many hosts that have updated /etc/passwd during a series of system reconfi guration events, the inclusion of such fi les in the feature vector space reduces the distances from abnormal points to normal points, resulting in higher false negative rates. We discuss this further in Section 5.

\subsection{Real Attacks}

Now we proceed to examine the effi cacy of Seurat during a real worm outbreak. The best way to show this would be to have Seurat detect an anomaly caused by a new worm propagation. Instead of waiting for a new worm's outbreak, we have set up an isolated computer cluster where, without damaging the real network, we can launch worms and record fil le system changes. This way, we have full control over the number of hosts infected, and can repeat the experiments. Because the isolated cluster has no real users, we merge the data acquired from the isolated cluster with the data we have collected from the teaching cluster in order to conduct experiments.

We obtained the binaries and source codes of a few popular worms from public Web sites such as whitehats [18] and packetstorm [19]. Extensively testing Seurat, with various real worms in the isolated cluster, requires tremendous effort in setting up each host with the right versions of vulnerable software. As a fi rst step, we show the result with the Lion worm [20] in this experiment.

The Lion worm was found in early 2001. Lion exploits a vulnerability of BIND 8.2, 8.2-P1, 8.2.1, 8.2.2-Px. Once Lion infects a system, it sets up backdoors, leaks out confi dential information (/etc/passwd, /etc/shadow) via email, and scans the Internet to recruit vulnerable systems. Lion scans the network by randomly picking the fi rst 16 bits of an IP address, and then sequentially probing all the $2^{16}$ IP addresses in the space of the block. After that, Lion randomly selects another such address block to continue scanning. As a result, once a host is infected by Lion, all the vulnerable hosts nearby (in the same IP address block) will be infected soon. Lion affects fi le systems: the worm puts related binaries and shell scripts under the / dev/. l ib directory, copies itself into the /tmp directory, changes system fi les under the /etc directory, and tries to wipe out some $\log$ fi les. 
We confi gured the isolated cluster with three Lion-vulnerable hosts and one additional machine that launched the worm. The vulnerable machines were running RedHat 6.2 including the vulnerable BIND 8.2.2-P5. The cluster used one C class network address block. Every machine in the cluster was connected to a $100 \mathrm{Mbps}$ Ethernet and was running named. The Seurat data collection tool generated a fi le system update report on every machine daily.
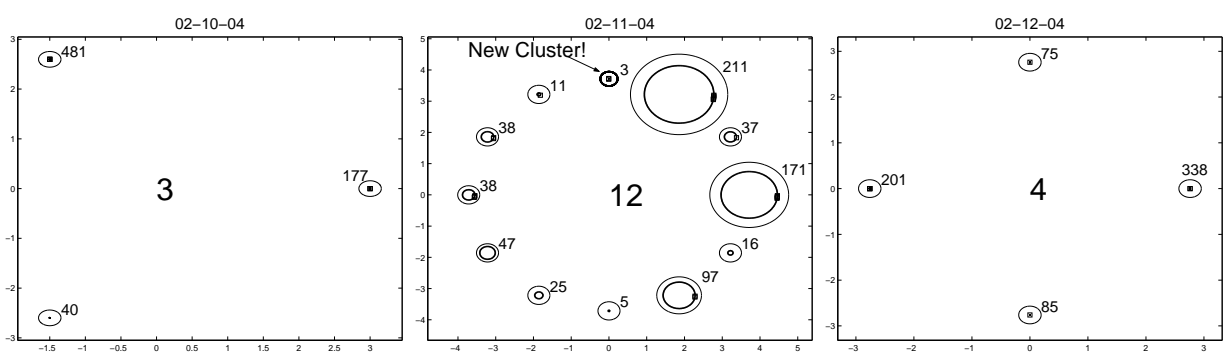

Fig. 11. Intrusion detection by Seurat: Seurat identified a new cluster of three hosts on Feb 11, 2004, when we manually launched the Lion worm

After we launched the Lion worm, all three vulnerable hosts in the isolated cluster were infected quickly one after another. We merge the fi le update report by the each compromised host with a different normal host report generated on Feb 11, 2004, when we know there was no anomaly. Figure 11 shows the clustering results of three consecutive days from Feb 10, 2004 to Feb 12, 2004 using the merged reports.

On the attack day, there are 64 fi les picked by the wavelet-based selection. The number of feature dimensions is reduced to 9 after PCA. Seurat successfully detects a new cluster consisting of the 3 infected hosts. Figure 12 lists the 22 fi les selected by Seurat as the causes of the alarm. These fi les provide enough hints to the administrators to confi rm the existence of the Lion worm. Once detected, these compromised hosts as well as the list of suspicious fi les can be marked for future detection. If, in the following days, there are more hosts that are clustered together with the already infected machines, or experience the same fi le updates, then we may conclude they are infected by the same attack.

\section{Discussion}

\subsection{Vulnerabilities and Limitations}

By identifying parallel occurrences of coincident events, Seurat will be most successful in detecting virus or worm propagations that result in fi le modifi cations at multiple hosts. Certain attacks (e.g., password guessing attacks) that succeed only once or a few times in a network system may evade Seurat detection. The current prototype of Seurat also has limited detection capability to the following types of attacks. 


\begin{tabular}{|c|c|c|c|}
\hline File ID. & File name & File ID. & File name \\
\hline 1 & $/ \mathrm{sbin} / \mathrm{asp}$ & 12 & /var/spool/mail \\
\hline 2 & $/ \mathrm{dev} / .1 \mathrm{ib}$ & 13 & /dev/.lib/bindx.sh \\
\hline 3 & /dev/.lib/star.sh & 14 & /tmp/ramen.tgz \\
\hline 4 & /var/spool/mail/root & 15 & /dev/.lib/scan.sh \\
\hline 5 & $/$ dev/.lib/bind & 16 & /dev/.lib/pscan \\
\hline 6 & /etc/hosts.deny & 17 & /var/spool/mqueue \\
\hline 7 & /dev/.lib/randb & 18 & /dev/.lib/hack.sh \\
\hline 8 & $/ \operatorname{sbin}$ & 19 & /dev/.lib/.hack \\
\hline 9 & /var/log & 20 & /dev/.lib/index.html \\
\hline 10 & /dev/.lib/bindname.log & 21 & /dev/.1ib/asp62 \\
\hline 11 & /dev/.lib/index.htm & 22 & /var/log/sendmail.st \\
\hline
\end{tabular}

Fig. 12. Suspicious files for the new cluster on Feb 11, 2004

Stealthy attack. Attackers may try to evade detection by slowing attack propagation. If an attacker is patient enough to infect only one host a day in the monitored network system, Seurat will not notice the intrusion with the current one-day detection window because Seurat focuses only on anomalous fi le changes common across multiple hosts. A larger detection window such as a couple of days or a week can help to catch slow, stealthy attacks. Note, however, that Seurat notices the attacks only after multiple hosts in the network system are compromised. In other words, if an attack propagates slowly, Seurat may not recognize the attack for the fi rst few days after the initial successful compromise. There is thus a tradeoff between detection rate and detection latency.

Mimicry attack. An attacker can carefully design his attack to cause fi le updates that look similar to regular fi le changes, and mount a successful mimicry attack [21]. There are two ways to achieve a mimicry attack against the current prototype. First, an attacker may try to fool Seurat's feature selection process by camouflaging all intrusion fi les as frequently, regularly updated fi les. Those concealed fi les, even when they are modifi ed in an unexpected way (e.g., entries removed from append-only log fi les), will not be selected as feature vector dimensions because of current use of the binary feature representation. Note that Seurat's data collection tool provides additional information on fi le system changes, such as fi le size, fi le content digest, and permissions. By incorporating the extra information in representing host state transition, Seurat can make such mimicry attacks harder. Second, an attacker may fi nd a way to cloak abnormal fi le updates with many normal but irregular changes during Seurat's clustering process. For example, in Section 4.2, we observed that the false negative rate of detecting the Kork worm was relatively higher due to the interference of irregular system reconfi guration. We leave it as future work to quantify this type of mimicry attack and the effectiveness of possible counter measures.

Random-file-access attack. Seurat correlates fi le updates based on their complete path names. Thus attackers can try to evade Seurat by installing attack fi les under different directories at different hosts, or replacing randomly chosen existing fi les with attack fi les. Many recent email viruses already change the virus fi le names when they propagate to a new host; we envision similar techniques could be employed by other 
types of attacks soon. Note, however, that even the random-fi le-access attack may need a few anchor fi les at fi xed places, where Seurat still has the opportunity to detect such attacks. A more robust representation of a fi le, for example, an MD5 checksum, could help Seurat detect random-fi le-access attacks.

Memory-resident attack. Memory-resident and BIOS-resident only attacks make no fi le system updates. Thus Seurat will not be able to detect memory resident attacks by examining host fi le updates, nor those attacks that erase disk evidence before the Seurat data collection tool performs the scheduled disk scan.

Kernel/Seurat modification attack. The effectiveness of Seurat relies on the correctness of reports from the data collecting tools running on distributed hosts. So the host kernels and the Seurat data collection tools should run on machines protected by trusted computing platforms [22]. An alternative solution is to monitor fi le system changes in real time (will be discussed further in Section 5.2) and to protect fi le update logs using secure audit logging [23].

\subsection{Future Work}

Real-time anomaly detection. The current prototype periodically scans and reports fi le system updates with a 1-day cycle, which may be slow to detect fast propagating attacks. To shorten detection latency, we are enhancing the Seurat data collection module to monitor system calls related with fi le updates, and report the changes immediately to the correlation module. The reported fi le updates will be instantly reflected by setting the corresponding bits in the feature vectors at the Seurat correlation module, which continuously performs clustering of the new feature vectors for real time anomaly detection.

Distributed correlation module. Currently, Seurat moves the daily reports from distributed data collection tools to a centralized server, where the correlation module computes and clusters the host vectors. Despite the simplicity of centralized deployment, the centralized approach exposes Seurat to problems in scalability and reliability. First, the amount of report data to be transferred to and stored at the centralized server is large. In our experience, a host generates a fi le update report of $3 \mathrm{~K}-140 \mathrm{KBy}$ tes daily in a compressed format, so the aggregate report size from hundreds or thousands of hosts with a long comparison window will be large. The report size will be larger when Seurat's data collection tool reports the host state changes in real time. Second, the monitored hosts could be in different administrative domains (i.e., hosts managed by different academic departments or labs) and it is often impractical to transfer the detailed reports from all the hosts to one centralized server due to privacy and confi dentiality issues. Third, the centralized server can be a single point-of-failure. It is important for Seurat to work even when one correlation server is broken or a part of network is partitioned. A distributed correlation module will cope with those issues. We are now investigating methods to correlate fi le update events in a distributed architecture such as EMERALD [1], AAFID [24], and Mingle [25].

Other applications. The approach of clustering coincident host state changes can be generalized to other types of applications such as detecting the propagation of spyware, illegal fi le sharing events, or erroneous software confi guration changes. We are currently 
deploying Seurat on Planetlab [26] hosts for detecting software confi guration errors by identifying host state vectors that do not fall into an expected cluster.

\section{Related Work}

Seurat uses fi le system updates to represent a host state change. File system updates have been known to be useful information for intrusion detection. Tripwire [4], AIDE [8], Samhain [27] are well-known intrusion detection systems that use fi le system updates to fi nd intrusions. Recently proposed systems such as the storage-based intrusion detection systems [7] and some commercial tools [28] support real-time integrity checking. All of them rely on a pre-defi ned rule set to detect anomalous integrity violation, while Seurat diagnoses the anomaly using learning and correlation across time and space.

Leveraging the information gathered from distributed multiple measurement points is not a new approach. Many researchers have noticed the potential of the collective approaches for intrusion detection or anomaly detection. Graph-based Intrusion Detection System (GrIDS) [29] detects intrusions by building a graph representation of network activity based on the report from all the hosts in a network. Different from Seurat, GrIDS uses the TCP/IP network activity between hosts in the network to infer patterns of intrusive or hostile activities based on pre-defi ned rules. Other systems, such as Cooperative Security Managers (CSM) [30], Distributed Intrusion Detection System (DIDS) [31], also take advantage of a collective approach to intrusion detection. They orchestrate multiple monitors watching multiple network links and track user activity across multiple machines.

EMERALD (Event Monitoring Enabling Responses to Anomalous Live Disturbances) [1] and Autonomous Agents For Intrusion Detection (AAFID) [24] have independently proposed distributed architectures for intrusion detection and response capability. Both of them use local monitors or agents to collect interesting events and anomaly reports (from a variety of sources; audit data, network packet traces, SNMP traffi c, application logs, etc.). The architectures provide the communication methods to exchange the locally detected information and an easy way to manage components of the systems. AAFID performs statistical profi le-based anomaly detection and EMERALD supports a signature-based misuse analysis in addition to the profi le-based anomaly detection. Note that Seurat starts with similar motivation. But Seurat focuses more on the technique for correlating the collective reports for anomaly detection, and infers interesting information on the system state from learning, rather than relying on a predefi ned set of events or rules. We envision Seurat as a complementary technique, not as a replacement of the existing architectures that provide global observation sharing.

Correlating different types of audit logs and measurement reports is another active area in security research. Many researchers have proposed to correlate multiple heterogeneous sensors to improve the accuracy of alarms [3,32,33,2,34]. In this work, we attempt to correlate information gathered by homogeneous monitors (especially, the fi le system change monitors) but we may enhance our work to include different type of measurement data to represent individual host status.

Wang et al. [35] also have noticed the value of spatial correlation of multiple system confi gurations and applied a collective approach to tackle misconfi guration trouble 
shooting problems. In their system, a malfunctioning machine can diagnose its problem by collecting system confi guration information from other similar and friendly hosts connected via a peer-to-peer network. The work does not target automatic detection of the anomaly, but rather it aims at fi guring out the cause of a detected problem.

\section{Conclusions}

In this paper, we presented a new "pointillist" approach for detecting aggregated anomalous events by correlating information about host fi le updates across both space and time. Our approach explores the temporal and spatial locality of system state changes through learning and correlation. It requires neither prior knowledge about normal host activities, nor system specifi c rules.

A prototype implementation, called Seurat, suggests that the approach is effective in detecting rapidly propagating attacks that modify host fi le systems. The detection rate degrades as the stealthiness of attacks increases. By trading off detection latency, we are also able to identify hosts that are compromised by slowly propagating attacks. For

each alarm, Seurat identifi es suspicious fi les and hosts for further investigation, greatly facilitating root cause diagnosis and false alarm suppression.

\section{References}

1. Porras, P.A., Neumann, P.G.: EMERALD: Event Monitoring Enabling Responses to Anomalous Live Disturbances. In: Proceedings of the 20th National Information Systems Security Conference. (1997)

2. Abad, C., Taylor, J., Sengul, C., Zhou, Y., Yurcik, W., Rowe, K.: Log Correlation for Intrusion Detection: A Proof of Concept. In: Proceedings of the 19th Annual Computer Security Applications Conference, Las Vegas, Nevada, USA (2003)

3. Kruegel, C., Toth, T., Kerer, C.: Decentralized Event Correlation for Intrusion Detection. In: International Conference on Information Security and Cryptology (ICISC). (2001)

4. Tripwire, Inc.: Tripwire. (http: //www.tripwire.com)

5. CERT Coordination Center: Overview of Attack Trends. http://www.cert.org/ archive/pdf/attack_trends.pdf (2002)

6. Moore, D., Paxson, V., Savage, S., Shannon, C., Staniford, S., Weaver, N.: Inside the Slammer Worm. IEEE Security and Privacy 1 (2003) 33-39

7. Pennington, A., Strunk, J., Griffin, J., Soules, C., Goodson, G., Ganger, G.: Storage-based intrusion detection: Watching storage activity for suspicious behavior. In: Proceedings of 12th USENIX Security Symposium, Washington, DC (2003)

8. Lehti, R., Virolainen, P.: AIDE - Advanced Intrusion Detection Environment. (http: //www.cs.tut.fi/ rammer/aide.html)

9. Berry, M.W., Drmac, Z., Jessup, E.R.: Matrices, vector spaces, and information retrieval. SIAM Review 41 (1999)

10. Kamber, M.: Data mining: Concepts and techniques. Morgan Kaufmann Publishers (2000)

11. Zhang, J., Tsui, F., Wagner, M.M., Hogan, W.R.: Detection of Outbreaks from Time Series Data Using Wavelet Transform. In: AMIA Fall Symp., Omni Press CD (2003) 748-752

12. Jolliffe, I.T.: Principle component analysis. Spring-Verlag, New York (1986)

13. Forgy, E.: Cluster analysis of multivariante data: Efficiency vs. Interpretability of classifications. Biometrics 21 (1965) 
14. Gersho, A., Gray, R.: Vector Quantization and Signal Compresssion. Kluwer Academic Publishers (1992)

15. Moore, A.: K-means and Hierarchical Clustering. http://www.cs . cmu . edu/ awm/ tutorials/kmeans09.pdf (available upon request) (2001)

16. Symantec: Symantec Security Response. (http://securityresponse. symantec. $\mathrm{com})$

17. F-Secure: F-Secure Security Information Center. (http://www.f-secure.com/ virus-info)

18. Whitehats, Inc.: Whitehats Network Security Resource. (http://www.whitehats. com)

19. PacketStorm: Packet Storm. (http://www.packetstormsecurity.org)

20. SANS Institute: Lion Worm. http://www. sans.org/y $2 \mathrm{k} / \mathrm{lion}$. htm (2001)

21. Wagner, D., Dean, D.: Mimicry Attacks on Host-Based Intrusion Detection Systems. In: Proceedings of ACM Conference on Computer and Communications Security (CCS). (2002)

22. Trusted Computing Platform Alliance: Trusted Computing Platform Alliance. (http: //www.trustedcomputing.org)

23. Schneier, B., Kelsey, J.: Cryptographic Support for Secure Logs on Untrusted Machines. In: The Seventh USENIX Security Symposium. (1998)

24. Balasubramaniyan, J.S., Garcia-Fernandez, J.O., Isacoff, D., Spafford, E., Zamboni, D.: An architecture for intrusion detection using autonomous agents. In: Proceedings of the 14th IEEE Computer Security Applications Conference. (1998)

25. Xie, Y., O'Hallaron, D.R., Reiter, M.K.: A Secure Distributed Search System. In: Proceedings of the 11th IEEE International Symposium on High Performance Distributed Computing. (2002)

26. Planetlab: PlanetLab. (http://www.planet-lab.org)

27. Samhain Labs: Samhain. (http://la-samhna.de/samhain)

28. Pedestal Software: INTACT ${ }^{\mathrm{TM}}$. (http://www.pedestalsoftware.com/ products/intact)

29. Cheung, S., Crawford, R., Dilger, M., Frank, J., Hoagland, J., Levitt, K., Rowe, J., StanifordChen, S., Yip, R., Zerkle, D.: The Design of GrIDS: A Graph-Based Intrusion Detection System. Technical Report CSE-99-2, U.C. Davis Computer Science Department (1999)

30. White, G., Fisch, E., Pooch, U.: Cooperating security managers: A peer-based intrusion detection system. IEEE Network 10 (1994)

31. Snapp, S.R., Smaha, S.E., Teal, D.M., Grance, T.: The DIDS (distributed intrusion detection system) prototype. In: the Summer USENIX Conference, San Antonio, Texas, USENIX Association (1992) 227-233

32. Valdes, A., Skinner, K.: Probabilistic Alert Correlation. In: Recent Advances in Intrusion Detection, Volume 2212 of Lecture Notes in Computer Science, Springer-Verlag (2001)

33. Andersson, D., Fong, M., Valdes, A.: Heterogeneous Sensor Correlation: A Case Study of Live Traffic Analysis. Presented at IEEE Information Assurance Workshop (2002)

34. Ning, P., Cui, Y., Reeves, D.S.: Analyzing Intensive Intrusion Alerts Via Correlation. In: Recent Advances in Intrusion Detection, Volume 2516 of Lecture Notes in Computer Science, Springer-Verlag (2002)

35. Wang, H., Hu, Y., Yuan, C., Zhang, Z.: Friends Troubleshooting Network: Towards PrivacyPreserving, Automatic Troubleshooting. In: Proceedings of the 3rd International Workshop on Peer-to-Peer Systems (IPTPS). (2004) 